\title{
GSK3 $\beta$ Hyperactivity during an Early Critical Period Impairs Prefrontal Synaptic Plasticity and Induces Lasting Deficits in Spine Morphology and Working Memory
}

\author{
Bo Xing ${ }^{1,2}$, Yan-Chun Li' and Wen-Jun Gao*,I \\ 'Department of Neurobiology \& Anatomy, Drexel University College of Medicine, Philadelphia, PA, USA; ${ }^{2}$ Xi'an Mental Health Center, Xi'an, \\ Shaanxi, PR China
}

\begin{abstract}
Schizophrenia (SZ) is a neurodevelopmental disorder in which the emergence of cognitive symptoms occurs during early adolescence. Glycogen synthase kinase-3 $\beta$ (GSK3 $\beta$ ) plays a critical role in synaptic plasticity during development and is highly implicated in the etiology of SZ. However, how GSK3 $\beta$ activity affects synaptic plasticity and working memory function in the prefrontal cortex (PFC) during development remains unknown. Here we show a GSK3 $\beta$ hyperactivity during the early postnatal period in a neurodevelopmental rat SZ model that receives gestational exposure (EI7) to the neurotoxin methylazoxymethanol (MAM). Accompanied with this change, adult MAM rats exhibited a significant decrease in spine density as well as impaired working memory, which was rescued by treatment with a GSK3 $\beta$ inhibitor during the juvenile period. Furthermore, the age-dependent hyperactive GSK3 $\beta$ caused a significant deficit in long-term potentiation (LTP) and facilitated long-term depression (LTD) in PFC pyramidal neurons. Notably, these changes in synaptic plasticity occurred only during the late juvenile period and were efficiently reversed by application of GSK3 $\beta$ inhibitors. Because the balance of LTP and LTD plays a critical role in activity-dependent synaptic stabilization and elimination during cortical development, the transient hyperactive GSK3 $\beta$ likely accounts for the cortical spine loss and PFC-dependent cognitive deficits in adulthood. These results highlight the importance of the postnatal trajectory of GSK3 $\beta$ for spine development and PFC function, and may shed light on the prophylactic treatment of cognitive symptoms in the SZ.

Neuropsychopharmacology (2016) 4I, 3003-30I5; doi:I0.I038/npp.20 I6.I I0; published online 20 July 2016
\end{abstract}

\section{INTRODUCTION}

Schizophrenia (SZ) is a neurodevelopmental disorder with cognitive deficits emerging in early stages of development (Insel, 2010). The specific molecular initiators of cognitive deficits remain unknown, but synaptic changes in the developing brain play an essential pathogenic role. Adolescent brain maturation is characterized by the emergence of executive function mediated by the prefrontal cortex (PFC) (Kolb et al, 2012). Overgrowth and pruning of excitatory synapses are signature morphological events of brain maturation during development, and reduction of dendritic spine density in the PFC is a hallmark pathological change in SZ (Bourgeois et al, 1994; Selemon, 2013). However, the mechanisms associated with the spine loss remain unclear. Mounting evidence suggests that synaptic plasticity, including both long-term potentiation (LTP) and long-term depression (LTD), is important for synaptic formation and elimination, respectively (Sala and Segal, 2014).

\footnotetext{
*Correspondence: Dr W-J Gao, Department of Neurobiology and Anatomy, Drexel University College of Medicine, 2900 Queen Lane, Room 243, Philadelphia, PA 19129, USA, Tel: + 215 991 8907, Fax: + I 215843 9802, E-mail: wgao@drexelmed.edu

Received 31 January 2016; revised 16 June 2016; accepted 21 June 2016; accepted article preview online 29 June 2016
}

Converging evidence suggests that aberrant serine phosphorylation in protein kinase glycogen synthase kinase 3 (GSK3) might be a common mechanism underlying the etiology of SZ (Emamian et al, 2004; Kozlovsky et al, 2002; Lovestone et al, 2007). There are two mammalian isoforms of GSK3, which are primarily regulated by inhibitory phosphorylation at either Ser21 of GSK3 $\alpha$ or Ser 9 for GSK3 $\beta$. Psychomimetic drugs increase GSK3 $\beta$ activity, whereas antipsychotics result in the inhibition of GSK $3 \beta$ by altering pGSK3 $\beta$ Ser9 levels (Freyberg et al, 2010; Li et al, 2007; Xi et al, 2011). Synaptic GSK3 $\beta$ has been demonstrated to be important in synaptic plasticity regulation, and crosstalk between LTP and LTD (Peineau et al, 2007). The activity of GSK3 $\beta$ is inhibited, as indicated by elevated inhibitory phosphorylation at Ser 9, during LTP induction (Zhu et al, 2007). Correspondingly, the elevated activity of GSK3 $\beta$, but not other serine/threonine kinases, seems to be required for LTD induction (Peineau et al, 2009,2007).

Notably, GSK3 $\beta$ has shown large fluctuations in its expression levels and phosphorylation states in juvenile and adolescent brains (Beurel et al, 2012; Leroy and Brion, 1999). In support of a developmental role for GSK3 $\beta$ in SZ etiology, reduced frontal cortical GSK3 $\beta$ levels are found in pre-pubertal rats with the neonatal excitotoxic hippocampal lesion (Nadri et al, 2003). However, how the aberrant GSK3 $\beta$ 
signal is associated with dendritic spine formation and elimination in SZ remains largely unknown. This question is of particular importance because PFC-dependent cognitive deficits in SZ usually emerge in early adolescence. Using the prenatal methylazoxymethanol acetate (MAM) exposure model of SZ, we provide compelling evidence that a transient GSK $3 \beta$ hyperactivity during late juvenile/early adolescence inhibits LTP and enhances LTD in prefrontal cortical synapses, leading to dendritic spine loss and working memory dysfunction, which could be rescued by early treatment with GSK3 $\beta$ inhibitors. Our results have both mechanistic and therapeutic implications for the initiation of prefrontal structural and functional deficits in SZ.

\section{MATERIALS AND METHODS}

Detailed methods can be found in the Supplementary Information. Briefly, all experiments were performed in accordance with the NIH Guidance and the procedures were approved by the IACUC of Drexel University. MAM rats were prepared as described previously (Snyder et al, 2013). The classification of age group (newborns on postnatal day (P1) to P7, early juvenile at P14, late juvenile at P21 to P28, adolescence at P45, and young adult at P60) was based on others' (Spear, 2000) and our previous studies (Snyder et al, 2013; Wang and Gao, 2009). Whole-cell extracts from medial PFC tissues were processed for western blot analysis with different antibodies as indicated in our published procedure (Xi et al, 2011).

Coronal slices $(300 \mu \mathrm{m})$ were prepared from PFC of SAL and MAM rats. Whole-cell patch clamp was obtained from visually identified layer $\mathrm{V}$ pyramidal cells in the prelimbic region of the PFC (Li et al, 2009; Snyder et al, 2013). Excitatory postsynaptic currents (EPSCs) were evoked by baseline stimulation using either a single pulse or a pairedpulse $(50 \mathrm{~ms}$ inter-pulse interval, $20 \mathrm{~Hz}$ ) at a frequency of $0.05 \mathrm{~Hz}$ with a holding potential of $-70 \mathrm{mV}$. To obtain the input/output (I/O) ratio, AMPAR-mediated EPSCs were elicited by a series of stimulation with incremental intensities $(0.5-1 \mathrm{~V})$. The LTP protocol used was presynaptic 80 pulses at $2 \mathrm{~Hz}$ paired with postsynaptic depolarization at $+30 \mathrm{mV}$, as previously reported (Hooper et al, 2007). For LTD induction, we used two protocols. The first one was a subthreshold protocol with 300 pairs of pulses at lowfrequency $(1 \mathrm{~Hz})$ stimulation (PP-LFS) at a $50-\mathrm{ms}$ inter-pulse interval (Caruana et al, 2011). The second one involved presynaptic 300 pulses at $1 \mathrm{~Hz}$ paired with postsynaptic depolarization at $-45 \mathrm{mV}$ (Toyoda et al, 2005). Picrotoxin $(100 \mu \mathrm{M})$ was always added into the bath solution to block $\mathrm{GABA}_{\mathrm{A}}$-mediated inhibitory currents. The GSK $3 \beta$ inhibitors, SB216763 and TDZD-8 (Tocris), were freshly prepared from a stock solution $(10 \mathrm{mM})$ by dissolving in dimethyl sulfoxide (DMSO) and bath-applied as indicated.

For some experiments, MAM or SAL rats were injected intraperitoneally with SB216763 (2 mg/kg/day; Tocris Bioscience) or vehicle (DMSO/Tween $80 / 0.9 \%$ saline) in a volume of $8 \mathrm{ml} / \mathrm{kg}$ for eight consecutive days (from P21 to P28). Assessments of Golgi-Cox staining and T-maze test were carried out using published methods when rats reached the age of P60 (see Supplementary Information). Two weeks after T-maze test, whole-cell current clamp recordings were used to record evoked EPSPs from the rats with different treatments (SAL-Vehicle, SAL-GSK3 inhibitors, MAM-Vehicle, and MAM-GSK3 inhibitors) in PFC layer $\mathrm{V}$ pyramidal neurons with $\mathrm{K}^{+}$-gluconate-based intracellular solution (Wang and Gao, 2009). Evoked EPSPs were elicited by a train of 10 stimuli delivered at $20 \mathrm{~Hz}$ with the membrane potentials of the recording cells adjusted to about $-70 \mathrm{mV}$ through a small holding current.

Data are expressed as mean \pm SEM. Student's $t$-test, oneway analysis of variance (ANOVA), two-way repeated measures ANOVA followed by Bonferroni post hoc test were used as noted. $P<0.05$ is used as a threshold for significance.

\section{RESULTS}

\section{GD17 MAM Exposure Induces an Increased GSK3 $\beta$ Activity in Developing PFC}

GSK $3 \beta$ is highly expressed throughout the brain with a dramatic fluctuation during early postnatal development in the hippocampus and neocortex (Beurel et al, 2012; Leroy and Brion, 1999). To determine the impact of GSK3 $\beta$ activity in the PFC during early postnatal development, we first examined the developmental expression of GSK3 $\beta$. Brain tissues containing the medial PFC (mPFC) were collected for western blotting at different ages. As shown in Figure 1a, protein levels of both total GSK3 $\beta$ (tGSK3 $\beta$ ) and GSK3 $\beta$ phosphorylation at Serine 9 (pGSK3 $\beta$ Ser9) peaked at P7 in the PFC, and then gradually decreased until adulthood (tGSK $3 \beta, \mathrm{F}=3.880, P<0.05 ; \mathrm{pGSK} 3 \beta, \mathrm{F}=7.520, P<0.001$ ). These results suggest that PFC development may be susceptible to alterations in GSK $3 \beta$ activity.

We also focused on the developmental changes of two other protein kinases, Akt1 and mTOR. Both are closely associated with GSK3 $\beta$ and SZ (Emamian et al, 2004). Akt1 is an upstream inhibitor of GSK3 $\beta$, while mTOR is an AKT downstream effector in the regulation of protein synthesis. Similar to the protein levels of tGSK3 $\beta$, total Akt1 levels rapidly declined from P7 to P45 ( $\mathrm{F}=27.60, P<0.001$; Figure $1 \mathrm{~b}$ ), but the pAktSer473/Akt1 ratios were relatively stable during development $(\mathrm{F}=1.159, P>0.05$; Figure $1 \mathrm{~b})$. In contrast, neither mTOR nor pmTORSer2448 showed developmental changes in the PFC (mTOR, F $=0.2201, P>0.05$; pmTORSer2448, $F=0.5029, P>0.05$; Figure 1c). These results suggest that activity of GSK3 $\beta$, but not Akt1 and mTOR, exhibits a significant fluctuation during postnatal development.

Next, using GD 17 MAM rats, we sought to determine the developmental trajectory of GSK3 $\beta$ activity. We observed a significant decrease in $\operatorname{pGSK} 3 \beta$ in the MAM group at P21 compared with that of saline (SAL) controls, although there was no change in the tGSK $3 \beta(P<0.05$; Figure $1 \mathrm{~d}$ and $\mathrm{e})$. At $\mathrm{P} 28$, the $\mathrm{pGSK} 3 \beta$ level in MAM rats remained significantly lower than that in SAL rats $(P<0.05$; Figure $1 \mathrm{f})$. Interestingly, no significant differences in the pGSK3 $\beta$ / $\operatorname{tGSK} 3 \beta$ ratios were found before and after the late juvenile (P21-P28) time points. Furthermore, we did not observe any significant changes in the ratios of pAkt/Akt1 and pmTOR/ mTOR between SAL and MAM rats during development (Figure 1d; P >0.05 for all MAM vs SAL, see Supplementary Information). Together, these data suggest that GD17 MAM exposure significantly increased postnatal GSK3 $\beta$ activity, indicated by the significant decrease in levels of inhibitory 
a

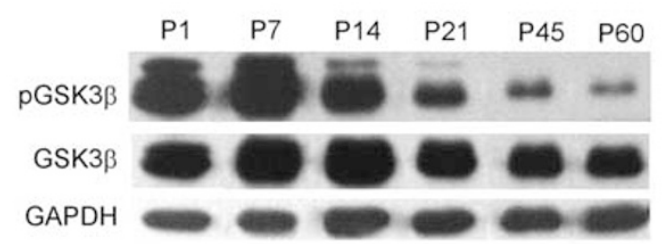

b
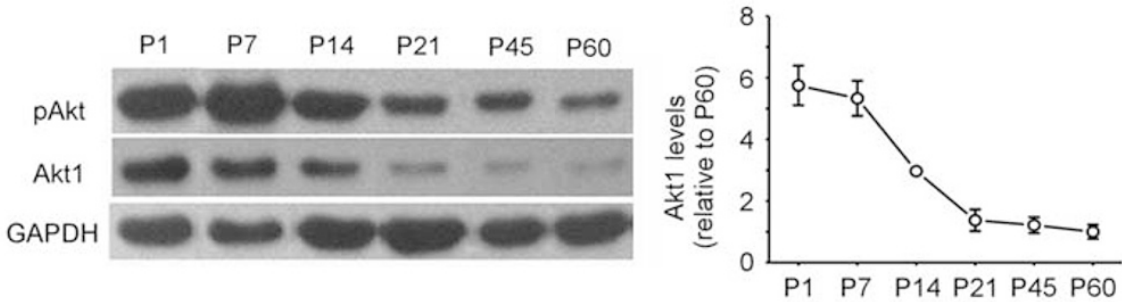

c
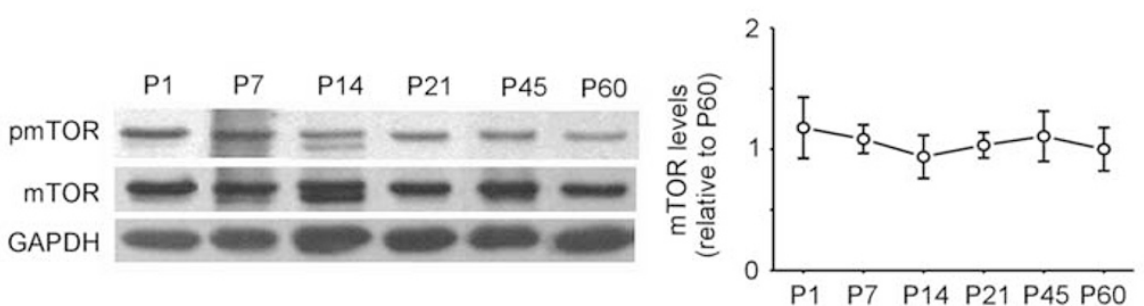
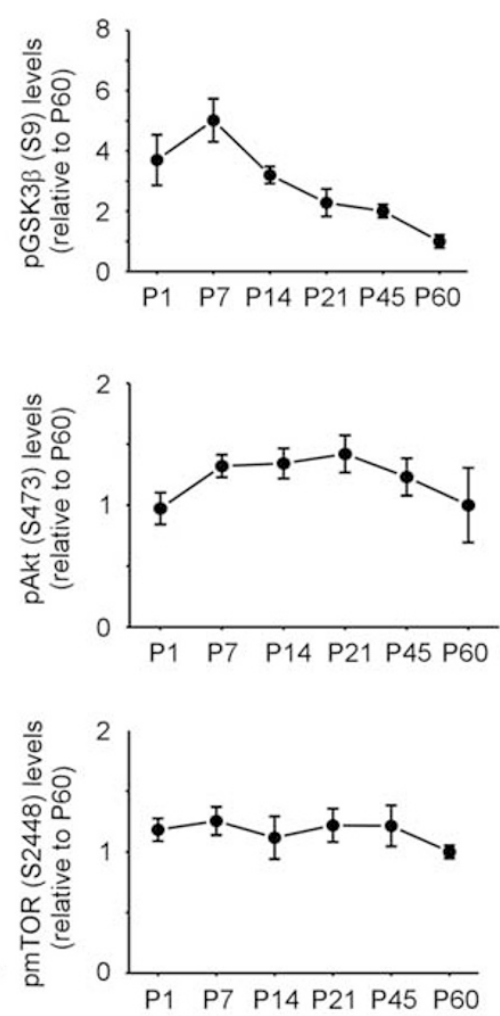

d
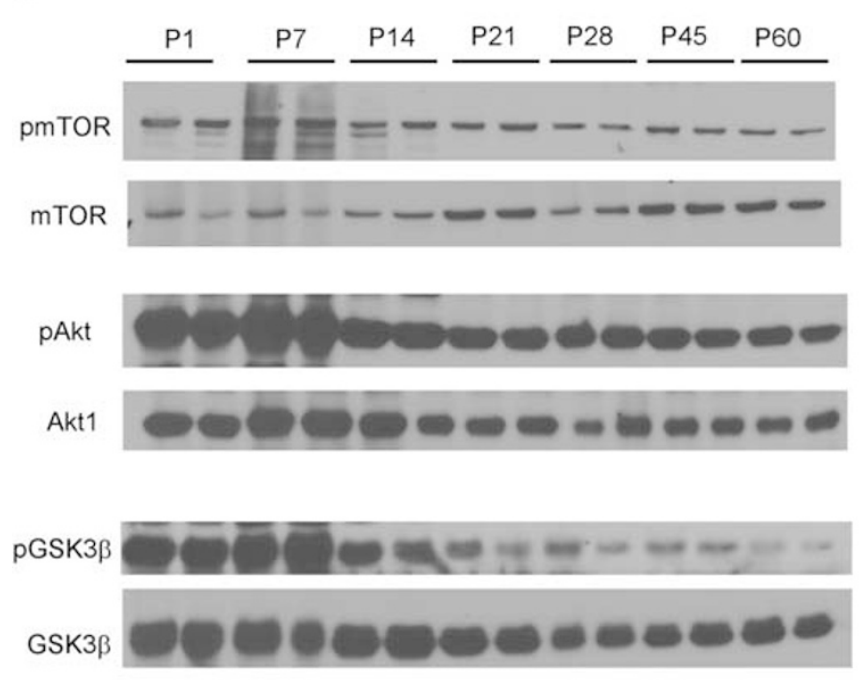

GAPDH

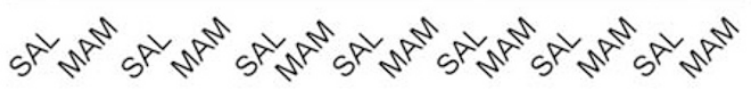

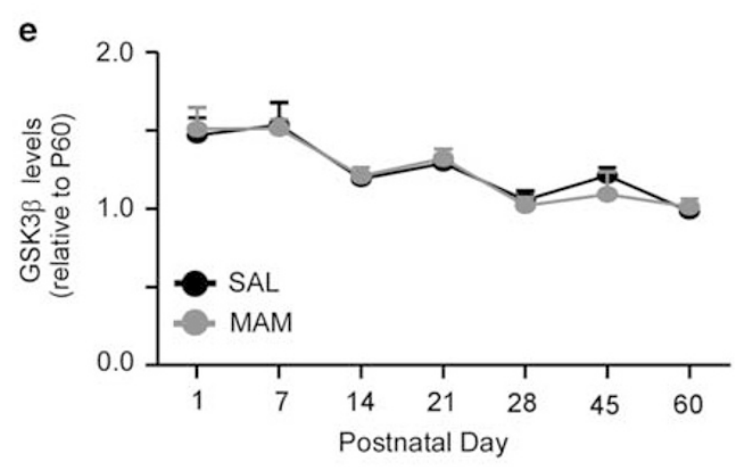

f

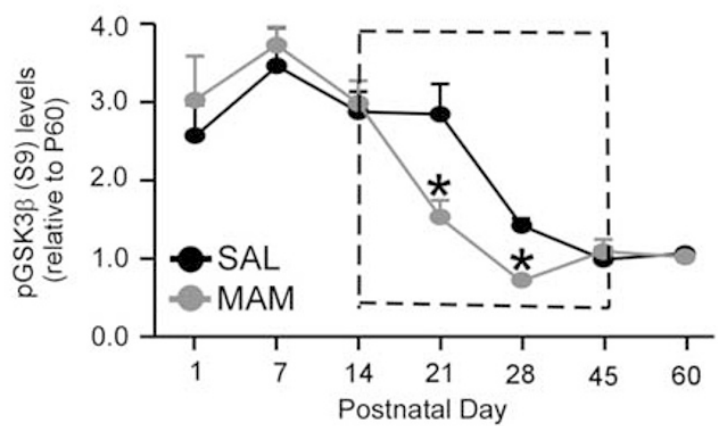

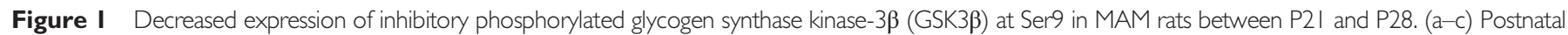
developmental profiles of the Akt/GSK3 $\beta / m T O R$ pathway in normal rat prefrontal cortex (PFC). Representative westem blots show that only the $p G S K 3 \beta / G S K 3 \beta$ ratio, but neither the pAktl/Akt I ratio nor the pmTOR/mTOR ratio, undergoes a rapid decrease during development. glyceraldehyde 3-phosphate dehydrogenase, which did not change during development, was used as a loading control. $n=6-10$ at each age. (d) Representative western blots show that pGSK3 $\beta / \mathrm{tGSK3} \beta$ ratios are significantly reduced in the PFC of MAM-exposed rats at P2I and P28 ( $n=6-12$ at each age), but not at PI, P7, PI4, P45, and P60. There were no significant differences in AktI, pAktI, mTOR, pmTOR at the age examined between SAL and MAM groups. (e, f) Summary graphs of tGSK3 $\beta$ and pGSK3 $\beta$ levels at different postnatal ages from SAL and MAM rats. The total protein levels of GSK3 $\beta$ are comparable between SAL and MAM rats in each age group (e). There were also no differences in the total protein levels of Akt I and mTOR between SAL and MAM rats (data not shown). Decreased ratios of pGSK3 $3 / t G S K 3 \beta$ were only observed in the MAM-exposed PFC during a restricted period of P2I-P28 (f, *P $<0.05$ vs SAL, Student's $t$-test). MAM, methylazoxymethanol. 
pGSK3 $\beta$ ser9, within a critical time window (late juvenile) in the developing PFC.

MAM Exposure Decreases Spine Number, while Developmental Inhibition of GSK3 $\beta$ Rescues Dendritic Spine Deficits in MAM-Exposed Rat PFC

One of the most replicated findings in SZ research is a reduced spine density on prefrontal cortical pyramidal neurons (Glantz and Lewis, 2000; Glausier and Lewis, 2013). Because of the critical role of GSK3 activity in synaptogenesis and spinogenesis (Cuesto et al, 2011), the aberrant GSK $3 \beta$ activity in developing PFC may underlie the dendritic spine deficits in SZ. We, therefore, asked whether GD17 MAM exposure decreased dendritic spine number in pyramidal neurons of the prelimbic region of $\mathrm{mPFC}$ in adults and whether treatment with a GSK $3 \beta$ inhibitor during the critical period could ameliorate dendritic spine deterioration. a

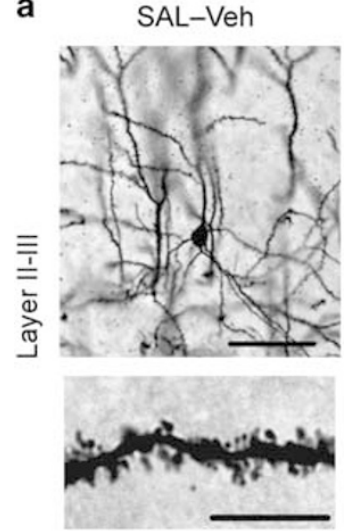

b
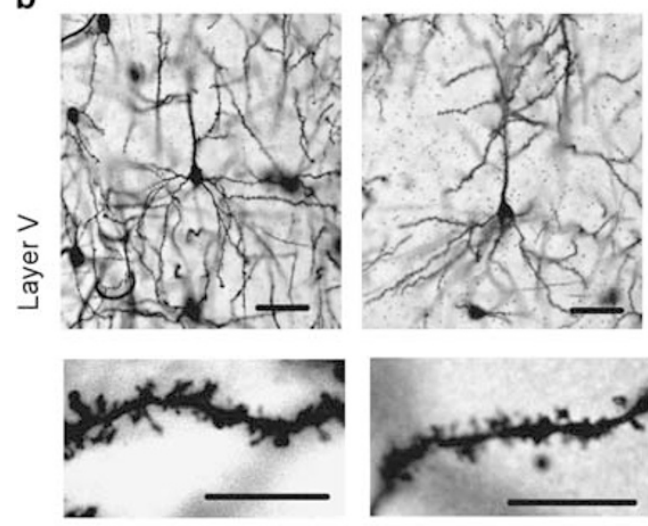

C

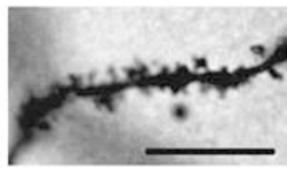
Layer II-III
apical dendrites

Layer V apical dendrites
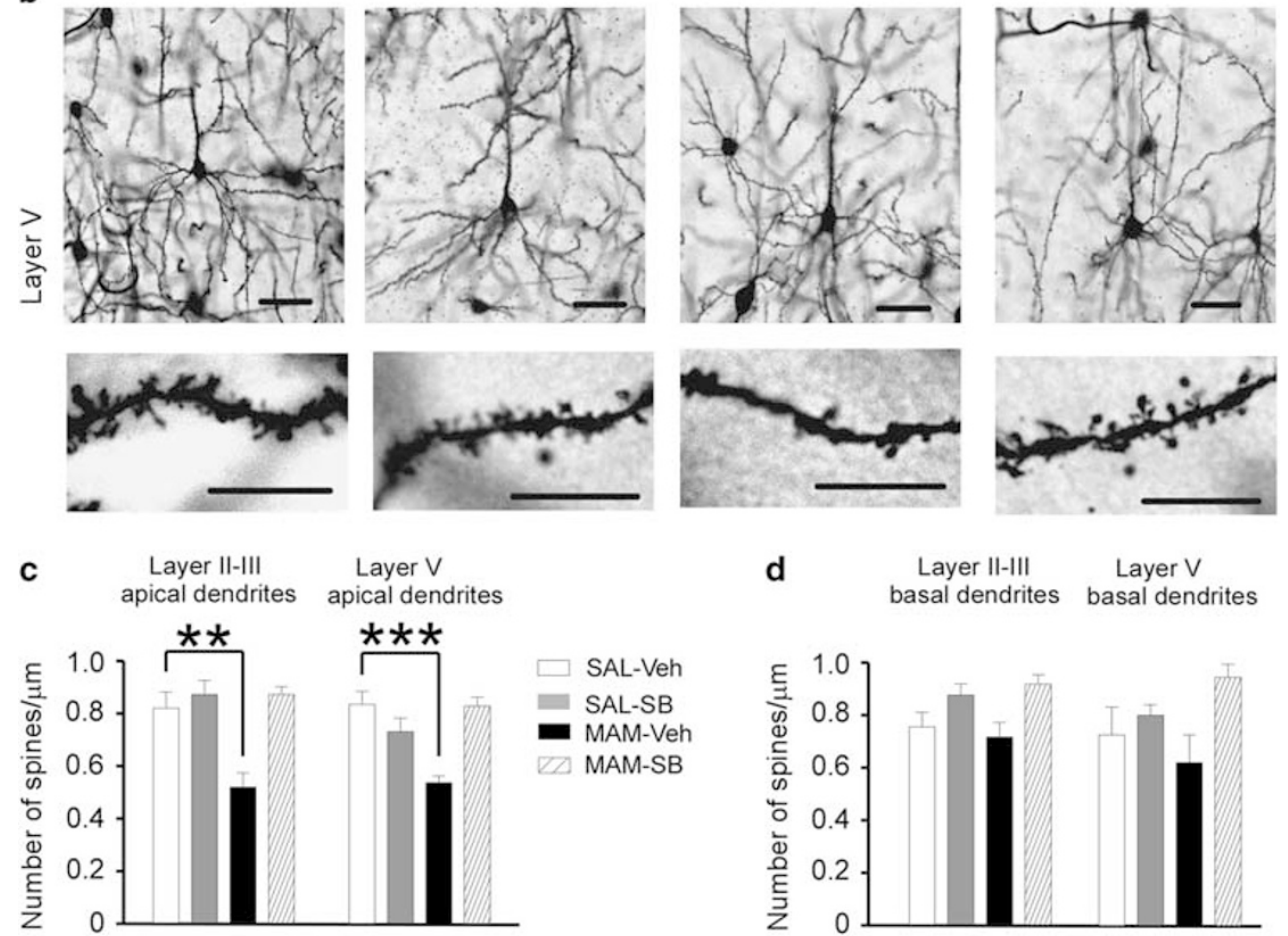

d

Layer II-III

Layer V basal dendrites basal dendrites

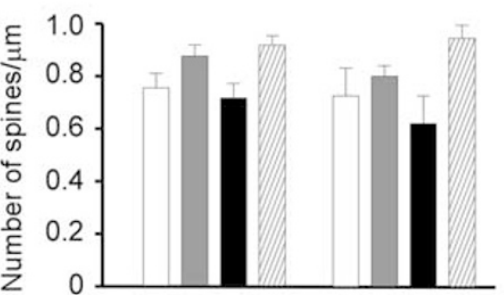

e

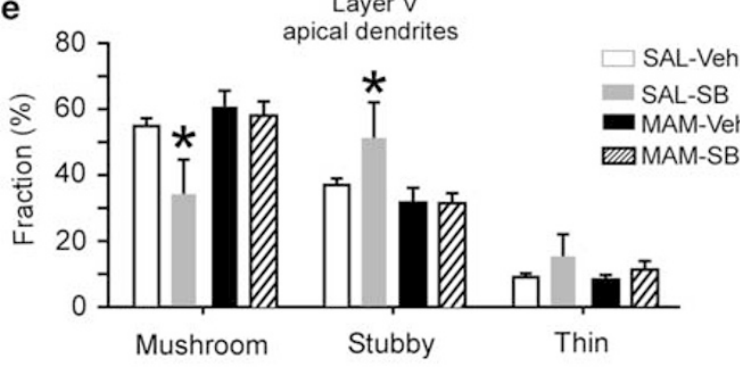


To test this idea, we counted the dendritic spine numbers in individual layers II-III and layer V pyramidal neurons in the PFC of SAL- and MAM-treated animals at P60 using the Golgi-Cox method with or without repeated administration of the selective GSK3 inhibitor, SB216763 (2 mg/kg/day, once a day), during P21-P28. As shown in Figure 2, MAM exposure significantly reduced apical dendritic spine density in both layers II-III $(0.51 \pm 0.05)$ and layer V $(0.53 \pm 0.03)$ neurons when compared with SAL controls (layers II-III $0.81 \pm 0.06$; layer V $0.83 \pm 0.05 ; P<0.01$ for both; Figure $2 \mathrm{a}-\mathrm{d})$ in the PFC of young adult rats (P60). Notably, in both layers II-III and layer V pyramidal neurons we found slight decreases but no statistical difference in basal dendritic spine density in MAM vs SAL animals $(P>0.05$ for both), indicating apical dendrites of pyramidal neurons were mainly affected in this animal model. More importantly, SB216763 treatment during P21-P28 significantly reversed the spine density loss in apical dendrites in MAM rats to comparable levels of SAL-Veh controls (layers II-III $0.87 \pm 0.03$; layer V $0.82 \pm 0.03, P>0.05$ vs SAL-Veh for both groups), while SB216763 itself did not show an obvious effect on SAL controls (layers II-III $0.87 \pm 0.05$; layer V $0.73 \pm 0.05$, $P>0.05$ vs SAL-Veh for both groups; Figure 2a-d). Again, there were no differences in the basal dendritic spine density among groups.

Next, we further analyzed spine morphology from the apical dendrites of the layer V pyramidal neurons. Dendritic spines were classified as mushroom, stubby, and thin spines, as previous studies reported (Peters and KaisermanAbramof, 1970). Unexpectedly, we observed a significant reduction of mushroom spines, an increase of stubby spines, and no change in thin spines in the apical dendrites of layer $\mathrm{V}$ pyramidal neurons of the SAL-SB group compared with those in the SAL-Veh control group (SAL-Veh, $n=6$; SAL-SB, $n=10 ; \quad$ MAM-Veh, $n=8 ;$ MAM-SB, $n=10$; $P<0.05$ vs SAL-Veh; Figure 2e). In contrast, the spine morphologies in the apical dendrites of PFC layer $\mathrm{V}$ neurons from both MAM-Veh and MAM-SB are similar to those in SAL controls (Figure 2e). This finding suggested that decreased GSK3 activity during the juvenile period may disrupt spine maturation in SAL control animals.

Together, these results suggested that prenatal MAM exposure produces spine loss specifically in apical dendrites of PFC pyramidal neurons, similar to those seen in postmortem findings in a subject with SZ (Glantz and Lewis, 2000). Furthermore, treatment with a GSK3 $\beta$ inhibitor during juvenile (P21-P28) effectively rescues these spine deficits in MAM-exposed rats, but disrupts spine maturation in SAL animals.

\section{Prefrontal Cortical LTP Deficit is Accompanied by Decreased pGSK3 $\beta$ Level in Juvenile MAM Rats}

The balance of glutamate receptor-mediated LTP and LTD influences the dynamic synaptic connections established during postnatal development, with GSK3 $\beta$ playing an integral regulator of both LTP and LTD (Hooper et al, 2007; Peineau et al, 2007; Zhu et al, 2007). This suggests that GSK $3 \beta$ may serve as a determinant of spine fate by modulating synaptic plasticity in vivo. To test this possibility, we investigated the role of GSK3 $\beta$ in LTP of excitatory synaptic transmission in the SAL and MAM rat mPFC. Recordings were collected in layer $\mathrm{V}$ pyramidal cells with stimulation of layer II/III to layer $\mathrm{V}$ pathway in acute prefrontal cortical slices (Supplementary Figure S1A). We found that the baseline strengths of AMPA-EPSCs in the MAM group showed no difference compared with those in SAL controls and the $\mathrm{I} / \mathrm{O}$ ratios in both groups were comparable during P21-P28 $(P=0.84$, two-way repeated-measures ANOVA, Saline $n=13$, MAM $n=15$; Supplementary Figure S1B). After a stable baseline recording, LTP was induced with a previously reported pairing LTP protocol (Hooper et al, 2007). We found that the magnitude of LTP in slices from juvenile (P21-P28) MAM rats was significantly decreased compared with that of SAL rats (SAL: $184.5 \pm 9.6 \%$ of the baseline EPSC amplitude vs MAM: $136.4 \pm 12.3 \%$ of baseline EPSC amplitude, $P<0.05$; Figure 3a). Interestingly, the LTP deficit was effectively blocked by bath-applied GSK3 $\beta$ inhibitor SB216763 $(5 \mu \mathrm{M}$, SAL: $\quad 166.6 \pm 21.3 \%$ vs MAM: $167.7 \pm 23.4 \%, \quad P>0.05$; Figure 3b). These results confirmed an LTP deficit in MAM rat PFC, similar to those shown in the hippocampus (Sanderson et al, 2012; Snyder et al, 2013), and more importantly, demonstrated a hyperactive GSK3 $\beta$-dependent LTP deficit.

\section{Hyperactive GSK $3 \beta$ Facilitated Prefrontal LTD in Juvenile MAM Rats}

Next, we focused on prefrontal LTD induction, as this process is closely associated with spine elimination (Bastrikova et al, 2008; Nagerl et al, 2004; Wiegert and Oertner, 2013; Zhou et al, 2004). Because high pGSK3 $\beta$ level inhibits LTD (Peineau et al, 2007), we proposed that a

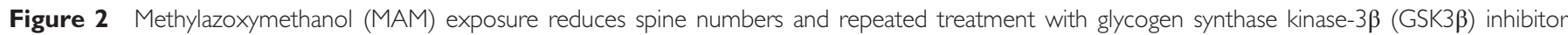
(SB2 I 6763) during the juvenile period (P2 I-P28) rescues spine density deficit in MAM rats at adulthood (P60). (a, b) Golgi-Cox-stained individual layers II-III

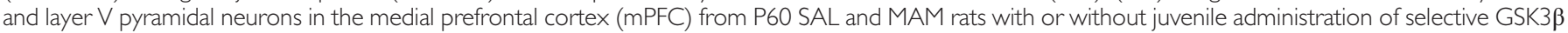
inhibitor SB2 I 6763 (2 mg/kg/day, i.p., once a day from P2I to P28). SAL-SAL, $n=6$ from 4 to 5 neurons; MAM-SAL, $n=7-10$ from 5 to 6 neurons; SAL-SB,

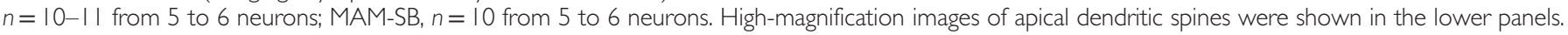
Scale bars $=50 \mu \mathrm{m}$ for the upper panel and $10 \mu \mathrm{m}$ for the lower panel. Pyramidal neurons in the layers $\|-I I I$ (a) show lower apical dendritic spine density in MAM rats vs the SAL controls. The treatment with SB2 6763 in juvenile restored spine density in the MAM rats. Similarly, Pyramidal neurons in layer $V$ (b)

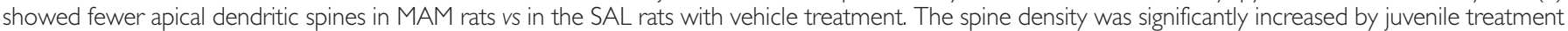

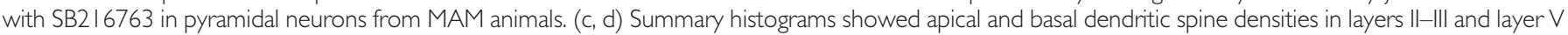

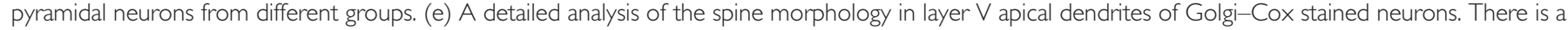

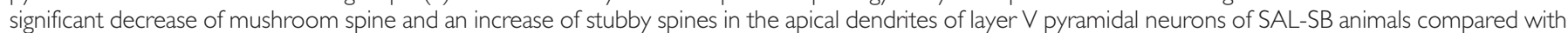

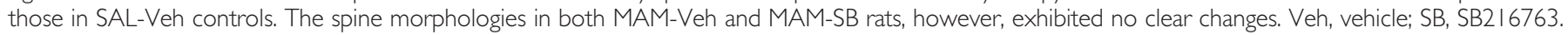
$* P<0.05$, ** $P<0.01$ and *** $P<0.001$ vs SAL-Veh, one-way ANOVA followed by Tukey's multiple comparison test. 
a
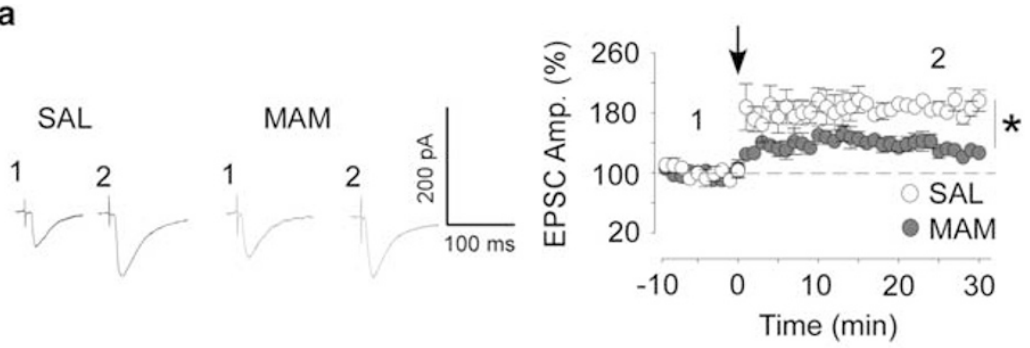

b
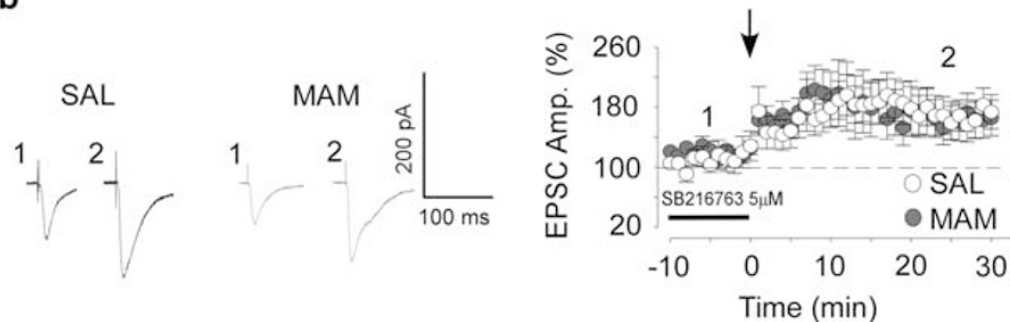

C

P21-P28
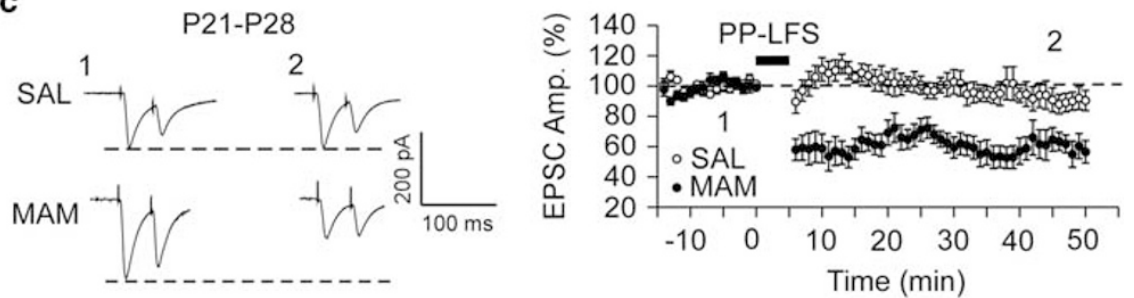

d

P45-P52
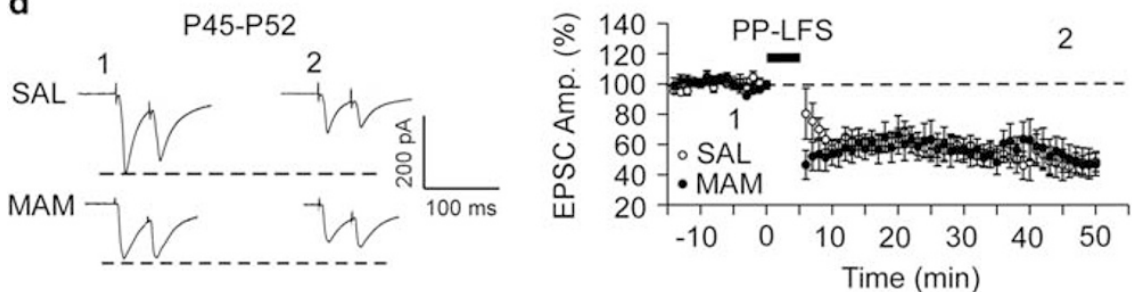

Figure 3 Long-term potentiation (LTP) deficit and long-term depression (LTD) facilitation in layer $V$ pyramidal neurons of juvenile MAM rats were reversed by SB2 I6763. (a) Summary plots of the magnitude of LTP induced by pairing protocol in prefrontal cortex (PFC) slices from juvenile (P2I-P28) SAL ( $n=6$ ) and MAM $(n=6)$ rats. In this and all subsequent LTP/LTD figures, representative excitatory postsynaptic current (EPSC) traces (average of I0 sweeps) were taken at the time indicated and calibration bars for the traces depict $200 \mathrm{pA}$ and $100 \mathrm{~ms}$. The arrow indicated the application of low-frequency pairing protocol. *P $<0.05$ vs SAL (Student's t-test). (b) A GSK-3 inhibitor (SB2I 6763, $5 \mu$ M, bath applied) reversed the LTP deficit in MAM rats ( $n=9$ ) without affecting the LTP induction in SAL rat PFC $(n=8)$. (c) 300 paired-pulse low-frequency stimulation (PP-LFS) induced significant LTD in layer $V$ pyramidal neurons of the PFC from juvenile (P2I-28) MAM rats $(n=9)$, but not from age-matched SAL rats $(n=8)$. The summary graph showed the normalized amplitude (Amp.) of evoked EPSCs during baseline and following PP-LFS. (d) 300 PP-LFS induced similar LTD in adolescent (P45-52) SAL ( $n=5$ ) and MAM $(n=6)$ rats. MAM, methylazoxymethanol.

decreased level of pGSK3 $\beta$ in the MAM rat PFC would facilitate the induction of LTD, which theoretically would promote excess synaptic pruning and spine loss during development. To test this hypothesis, we first investigated whether LTD could be induced in layer V pyramidal neurons of the mPFC. We also investigated whether LTD is developmentally regulated during the transition from juvenile to adolescence. We intentionally chose a stimulation protocol described in a previous study (Caruana et al, 2011) that would allow us to unmask the proposed LTD change in MAM-exposed animals. Specifically, evoked EPSCs were elicited at a holding potential of $-70 \mathrm{mV}$ in layer $\mathrm{V}$ pyramidal neurons from the $\mathrm{mPFC}$ by a paired-pulse $(20 \mathrm{~Hz}$ or $50 \mathrm{~ms}$ inter-stimulus interval) low-frequency stimulation (PP-LFS) of cortical layer I/II afferents. After $15 \mathrm{~min}$ of stable baseline recording, a protocol of $1 \mathrm{~Hz} 300$ PP-LFSs was applied. This protocol did not induce LTD in juvenile SAL-treated rats. Synaptic responses remained stable after the LFS $(99.3 \pm 2.9 \%, P>0.05$; Figure $3 c)$. In contrast, the same protocol produced a robust LTD in juvenile MAM rats $(60.8 \pm 5.9 \%, P<0.001$; Figure $3 c)$. Delivery of the protocol also induced a steady-state sustained LTD in both adolescent SAL group $(57.2 \pm 5.0 \%, P<0.001$; Figure $3 \mathrm{~d})$ and MAM rats $(55.1 \pm 8.2 \%, P<0.001$; Figure $3 \mathrm{~d})$. Together, these results suggested an age-dependent LTD facilitation in juvenile MAM rats. Notably, the LTD facilitation associated with juvenile MAM PFC was not mediated by the mechanisms from presynaptic release probability because 
a

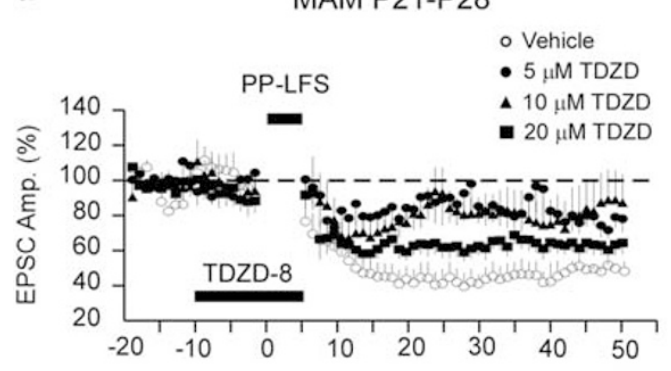

C

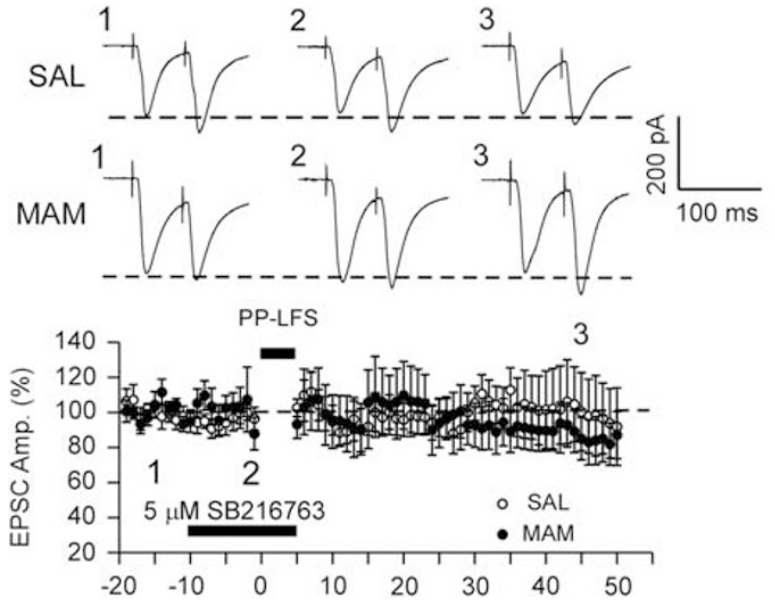

b

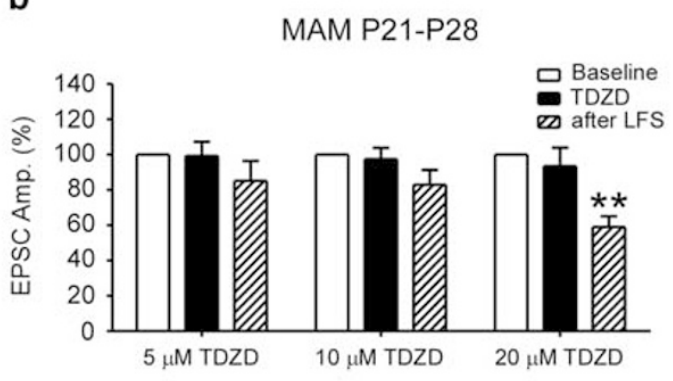

d

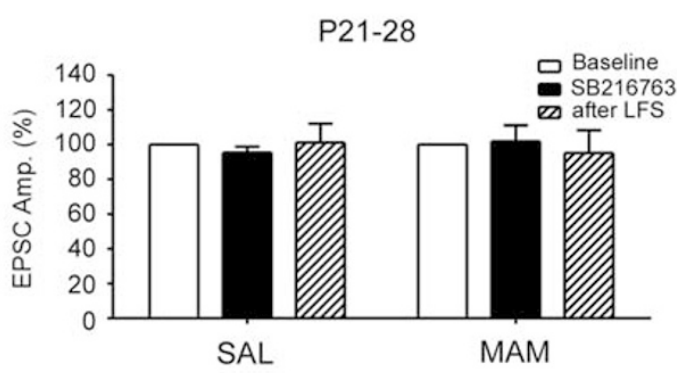

Figure 4 Glycogen synthase kinase-3 $\beta$ (GSK3 $\beta$ ) inhibitors blocked long-term depression (LTD) facilitation in the MAM rats. (a, b) GSK3 $\beta$ inhibitor TDZD-8 blocked LTD facilitation in the medial prefrontal cortex (mPFC) from juvenile (P2I-P28) MAM rats at lower doses. (a) Summary graph showed the normalized amplitudes of evoked excitatory postsynaptic current (EPSCs) in mPFC slices from MAM-exposed animals before, during, and after different doses of bath-applied TDZD-8 ( $\mu$ M, $5(n=7), 10(n=7)$, and $20(n=8)$ ). (b) Lower doses (5 and $10 \mu$ M) but not the higher dose (20 $\mu$ M) of TDZD-8 blocked LTD induction in juvenile MAM rats. **P $<0.01$ vs Baseline one-way ANOVA. (c, d) GSK3 $\beta$ inhibitor SB2 16763 completely blocked LTD facilitation in the mPFC of juvenile MAM rats $(n=8)$ with no effect on SAL controls $(n=8)$. (c) Evoked EPSCs in the mPFC layer $\vee$ pyramidal neurons did not change before, during, and after bath-application of SB216763 (5 $\mu$ M) in both SAL and MAM juvenile rats (P2I-P28). (d) Summary histogram showed that the amplitudes of evoked EPSCs in juvenile SAL- and MAM-exposed mPFC were comparable during baseline, SB216763 application, and the first and the last 5 min after 300 PP-LFS $(n=8, P<0.05$, one-way ANOVA). MAM, methylazoxymethanol.

we did not observe significant changes in the paired-pulse ratio (PPR) (Supplementary Figure S2).

LFS-induced LTD is associated with pGSK3 $\beta$ level decrease (hyperactive GSK3) (Peineau et al, 2007). In turn, the LTD facilitation could be a result of relatively lower levels of pGSK3 $\beta$ in juvenile MAM rats. To test this possibility, we collected prefrontal slices with or without stimulation and immediately determined the pGSK3 $\beta$ levels by western blotting. As shown in Supplementary Figure S3A and B, we found a significant decrease in pGSK $3 \beta$ levels in the MAM group compared with SAL control $(P<0.05)$ without PP-LFS, consistent with our prior data. Both SAL and MAM groups exhibited decreased pGSK3 $\beta$ after stimulation $(P<0.05)$, and the MAM-stimulation group had a lower but not significant level of pGSK3 $\beta$ compared with SAL-stimulation group $(P=0.11)$, indicating PP-LFS application reduced pGSK3 $\beta$, which may trigger LTD. Based on these findings, we hypothesized that stimulation-induced decrease of pGSK3 $\beta$ level in SAL control might not be sufficient to trigger LTD, whereas the relatively low basal pGSK3 $\beta$ level enables the MAM group to attain the LTD threshold. To test this, we applied a second 300 PP-LFS $10 \mathrm{~min}$ after the first stimulation protocol to SAL slices. We predicted that the initial PP-LFS-induced reduction in the pGSK3 $\beta$ level would facilitate LTD induction in response to a second stimulus. As expected, the first PP-LFS did not induce LTD in juvenile SAL-treated rat PFC neurons $(98.6 \pm 8.9 \%, P>0.05$; Supplementary Figure S3C and D). However, the second PP-LFS induced a robust and significant decrease of evoked EPSC that lasted for a minimum of $45 \min (48.1 \pm 6.8 \%, n=6 ; P<0.001$; Supplementary Figure S3C and D). Together, these results confirmed that a low pGSK3 $\beta$ level in MAM rats was the main contributor to the LTD facilitation during juvenile.

To further confirm that the LTD facilitation in MAM rats is GSK3 $\beta$ dependent, we examined the effects of two structurally distinct GSK3 $\beta$ inhibitors, TDZD-8 and SB216763. Since bath-applied vehicle (DMSO) for these drugs had no effect on LTD induction observed in juvenile MAM rats $(99.2 \pm 8.0 \%, P>0.05$ vs baseline; Figure $4 \mathrm{a})$, we investigated the effect of TDZD on LTD in these animals. At all dosages tested $(5,10$, and $20 \mu \mathrm{M})$, TDZD-8 had no significant effects on the amplitudes of baseline EPSCs (99.2 $\pm 8.0,97.5 \pm 6.3$, and $93.5 \pm 10.4 \%$, respectively, $P>0.05$ for all $v s$ baselines; Figure $4 \mathrm{a}$ and b). Combining 300 PP-LFS with TDZD-8 $(5 \mu \mathrm{M})$ resulted in a significant but partial 
block of LTD in the juvenile MAM group $(83.0 \pm 12.7 \%$, $P>0.05)$. TDZD-8 at $10 \mu \mathrm{M}$ produced a similar partial block of LTD induction $(79.4 \pm 8.6 \%, P>0.05)$. Unexpectedly, TDZD-8 lost its inhibitory effect on LTD induction at $20 \mu \mathrm{M}$ and the EPSCs remained depressed $(59.0 \pm 6.1 \%$, $P<0.01)$. We ascribed the failure of $20 \mu \mathrm{M}$ TDZD- 8 in the LTD block to a potential nonspecific off-target effect, as TDZD-8 at high concentration could exert an effect on other kinases, such as extracellular signal-regulated protein kinase (ERK), which is positively associated with LTD induction in the PFC. Specifically, the off-target effect such as ERK activation could lead to LTD induction, which in turn offset the effect of TDZD on LTD. This speculation, however, needs further exploration.

We next used SB216763 to further study the role of GSK3 $\beta$ in LTD in both juvenile and adolescent periods. Similarly, we did not observe any baseline change when SB216763 (5 $\mu \mathrm{M})$ was applied in juvenile rat layer $\mathrm{V}$ pyramidal neurons (SAL: $95.4 \pm 3.4 \%, P>0.05$ vs baseline response; MAM: $101.9 \pm 9.2 \%, P>0.05$; Figure $4 c$ and d). Pairing 300 PP-LFS with SB216763 $(5 \mu \mathrm{M})$ had no significant effect on the amplitude of evoked EPSC in the SAL control $(101.2 \pm 10.8 \%, \quad P>0.05$; Figure $4 \mathrm{c}$ and $\mathrm{d})$, whereas SB216763 completely blocked PP-LFS-induced LTD in the MAM rat PFC pyramidal neurons $(95.1 \pm 13.1 \%, P>0.05$; Figure $4 \mathrm{c}$ and $\mathrm{d}$ ). These results indicated that the GSK3 $\beta$ inhibitor, SB216763, effectively blocked the facilitation of LTD in juvenile MAM rats without an impact on basal synaptic transmission. Furthermore, SB216763 (5 $\mu \mathrm{M})$ also prevented LTD inductions in mPFC layer $\mathrm{V}$ pyramidal neurons of both adolescent SAL and MAM rats (SAL: $83.4 \pm 7.8 \%, \quad P>0.05$ vs baseline response; MAM: $89.5 \pm 6.9 \%, P>0.05$; Supplementary Figure S4A and B), suggesting a critical role of GSK $3 \beta$ activity in LTD induction during adolescent period.

We also measured PPRs before, during, and after SB216763 $(5 \mu \mathrm{M})$ application to determine whether presynaptic mechanisms were involved in LTD induction. The PPRs remained stable in the slices from juvenile MAM rats (Supplementary Figure S5A). These results indicated that postsynaptic mechanisms underlay the GSK3 $\beta$-mediated LTD facilitation. This conclusion was further confirmed by infusing SB216763 (5 $\mu \mathrm{M})$ into cells from juvenile MAM rats via recording electrodes to selectively block postsynaptic GSK3 $\beta$ activity. SB216763 infusion completely blocked the PP-LFS-induced LTD that was routinely observed in the juvenile MAM rats $(P>0.05$ vs vehicle control; Supplementary Figure S5B). Together, these data indicated that postsynaptic GSK3 $\beta$ was required to trigger LTD, consistent with a previous report (Peineau et al, 2007).

To probe whether the GSK $3 \beta$ was also involved in NMDAR-dependent LTD facilitation in MAM rats, we used a protocol pairing presynaptic stimulation (300 pulses at $1 \mathrm{~Hz}$ ) with postsynaptic depolarization (holding the membrane potential of recorded cell at $-45 \mathrm{mV}$ ). Given that inactivation of GSK $3 \beta$ blocked NMDAR-dependent LTD in a concentration-dependent manner (Peineau et al, 2007), we predicted hyperactivity of GSK $3 \beta$ in the MAM group would promote LTD. As expected, we found that NMDARdependent LTD was indeed enhanced in the PFC neurons of MAM rats compared with SAL control (Supplementary Figure S6), and LTD could be blocked by NMDA receptor antagonist $(R, S)$-CPP $(10 \mu \mathrm{M}$; Supplementary Figure S6). These results indicated that GSK $3 \beta$ hyperactivity was involved in NMDAR-dependent LTD facilitation in the MAM rats.

Finally, we determined whether treatment with SB216763 in vivo during the late juvenile period (P21-P28) was able to rescue LTP reduction and LTD facilitation in MAM-exposed rats. We found that SB216763 injection successfully rescued the LTP deficit in MAM rats $1 \mathrm{~h}$ after the last injection. SB216763 also slightly decreased the early stage of LTP, but had no significant effects overall on LTP induction, in SAL control animals (SAL-Veh: $161.7 \pm 9.5 \%$, SAL-SB: $160.9 \pm 9.3 \%$, MAM-Veh: $114.1 \pm 3.3 \%$, MAM-SB: $167.4 \pm 12.1 \%$; MAM-Veh vs SAL-Veh, $P<0.05$, MAM-SB vs SAL-Veh, $P>0.05$, Figure 5a-c). In addition, SB216763 treatment completely abolished LTD facilitation in MAM rats, but had no effects on LTD induction with PP-LFS in SAL controls (SAL-Veh: 98.1 $\pm 4.8 \%$, SAL-SB: $98.3 \pm 6.5 \%$, MAM-Veh: $44.7 \pm 4 . \quad 4 \%$, MAM-SB: $98.2 \pm 9.5 \%$; MAM-Veh vs SAL-Veh, $P<0.001$; MAM-SB vs SAL-Veh, $P>0.05$; Figure $5 \mathrm{~d}-\mathrm{f}$ ). These findings confirmed that treatment with SB216763 does have a significant effect on synaptic plasticity in vivo.

\section{Treatment of Juvenile MAM Rats with GSK3 $\beta$ Inhibitor Improved Working Memory}

To determine whether repeated treatment with a GSK3 $\beta$ inhibitor during the juvenile period could improve spatial working memory in MAM rats, we treated MAM rats with an i.p. injection of SB216763 (2 mg/ $\mathrm{kg} /$ day) or vehicle during the late juvenile period (P21-P28) with age-matched SAL rats as controls. A T-maze DNMTP task was employed in adulthood, starting from P60. Our results showed that there was no significant change in body weight during the drug administration period ( $P>0.05$; Supplementary Figure S7), and the training time required for criterion performance was comparable among all tested groups $(P>0.05$; Figure $6 a)$. However, in agreement with previous reports (Flagstad et al, 2005), MAM rats had an impaired performance in $30 \mathrm{~s}$ $(P<0.05$; Figure $6 \mathrm{~b})$, but not in other intratrial delays. Importantly, SB216763 treatment successfully rescued the impairments (Figure 6b). Surprisingly, SAL rats administered the GSK $3 \beta$ inhibitor during juvenile showed similar working memory deficits, as observed in MAM rats without SB216763 treatment $(P<0.05$; Figure 6b).

To assess the neuronal underpinnings of the behavioral impairments of the SAL rats with juvenile SB216763 treatment, we compared the temporal summation of glutamatergic synapses that strongly shape information transfer in cortical networks among groups. Experiments were conducted in PFC slices of rats 2 weeks after T-maze test with food and water available ad libitum. The membrane potentials of layer $\mathrm{V}$ pyramidal neurons were adjusted to $-70 \mathrm{mV}$, and 10 stimuli at $20 \mathrm{~Hz}(50 \mathrm{~ms}$ intervals $)$ were applied to layers I-II border. The peaks of evoked EPSPs were measured to determine the temporal summation of excitatory transmission. As shown in Figure $6 \mathrm{c}-\mathrm{g}$, in the groups with working memory deficits, including MAM-Veh and SAL-SB rats, the evoked EPSPs exhibited reduced synaptic summation, whereas in the SAL-Veh and MAM-SB groups, the eEPSPs showed an increased summation in response to the 10 -pulse stimuli. 
a

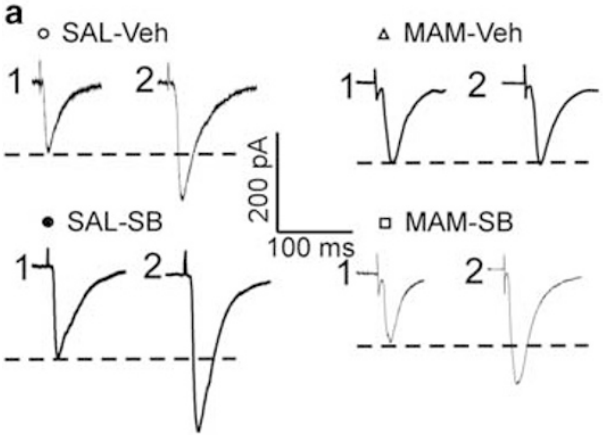

b
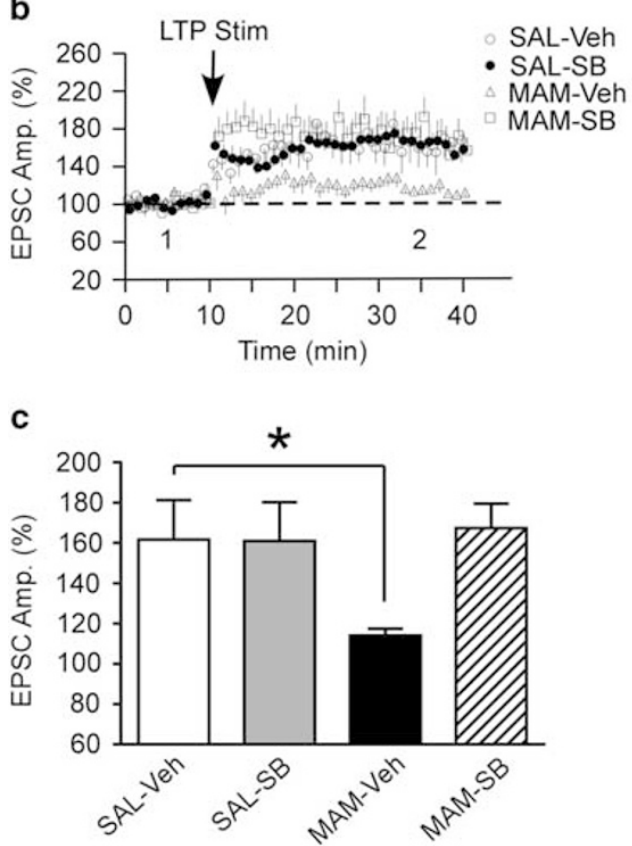

d OSAL-Veh

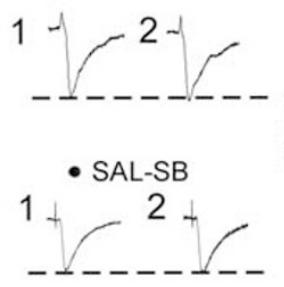

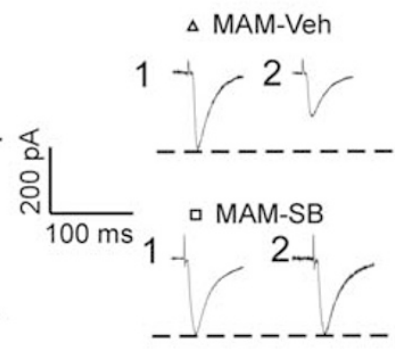

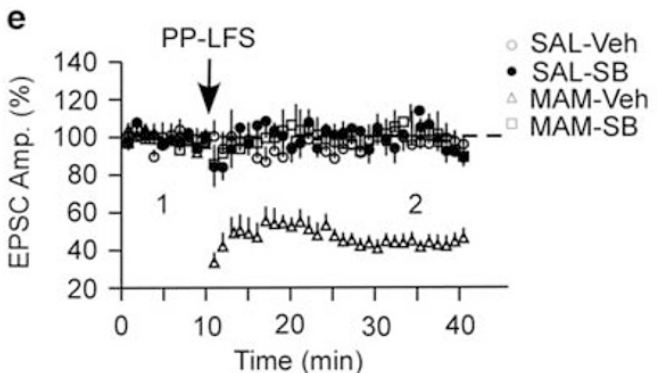

f

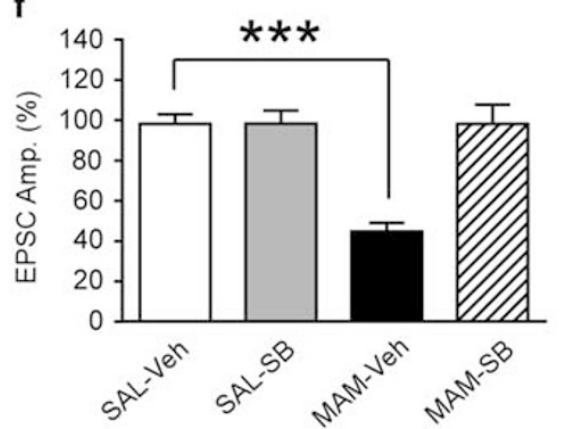

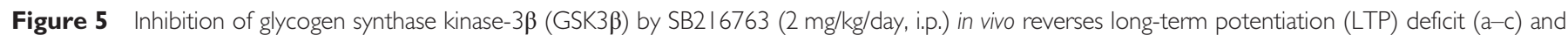
long-term depression (LTD) facilitation (d-f) in juvenile (P2I-P28) MAM rats. (a) Representative evoked excitatory postsynaptic current (EPSCs) recorded before and after LTP protocol application in SAL and MAM rats with Veh or SB2 16763 treatment. (b) Summary graph showed the normalized amplitude of eEPSCs from juvenile SAL-Veh $(n=8)$, SAL-SB $(n=8)$, MAM-Veh $(n=11)$, and MAM-SB $(n=8)$ rats during the LTP recording. (c) Summary histogram showed that LTP deficits in MAM rats can be rescued by SB21 6763 treatment. *P $<0.05$ vs SAL-Veh, one-way ANOVA. (d) Representative evoked EPSCs recorded before and after PP-LFS LTD protocol application in SAL and MAM rats with Veh or SB216763 injection. (e) Summary graph showed the normalized amplitude of eEPSCs from SAL-Veh $(n=8)$, SAL-SB $(n=8)$, MAM-Veh $(n=13)$, and MAM-SB $(n=10)$ groups during the LTD recording. (f) Summary histogram showed that LTD facilitation in MAM-Veh rats could be reversed by treatment with SB2 I 6763 . **** $P<0.00$ I vs SAL-Veh, one-way ANOVA. MAM, methylazoxymethanol.

\section{DISCUSSION}

We have explored the postnatal development of GSK3 $\beta$ in the PFC and found transiently hyperactive levels of GSK3 $\beta$ during the juvenile period in the MAM model of SZ. The GSK3 $\beta$ activity change is accompanied with a significant reduction in dendritic spines and working memory deficits. More importantly, treatment with a GSK3 $\beta$ inhibitor during the juvenile stage effectively rescued dendritic spine and working memory deficits in adult animals. In addition, we have provided solid evidence showing how GSK3 $\beta$ activity affected LTP/LTD balance in prefrontal cortical neurons, thus providing a mechanistic interpretation of how GSK3 $\beta$ hyperactivity affects synaptic instability and resultant spine loss in an animal model for SZ. Given that GSK3 is implicated in neuroplasticity deficits in SZ, elucidating its role in disease- and development-related synaptic impairment has important implications for therapeutic intervention. Overall, our data provide a novel insight into the role of GSK3 $\beta$ hyperactivity in early onset of cognitive deficits in SZ.

$\mathrm{SZ}$ is a neurodevelopmental disorder with largely unknown neuropathophysiology (Snyder et al, 2013). Although MAM exposure is not without controversy, as with many other animal models (Carpenter and Koenig, 2008), adult MAM-exposed animals display deficits similar to those seen in SZ patients, including neuroanatomical changes, behavioral deficits, and altered information processing (Lodge and Grace, 2009). Thus, this model reproduces some fundamental features of SZ with respect to both phenomenology and temporal pattern of the onset of symptoms, and provides a useful tool to explore the developmental origins of this disorder. To our knowledge, 

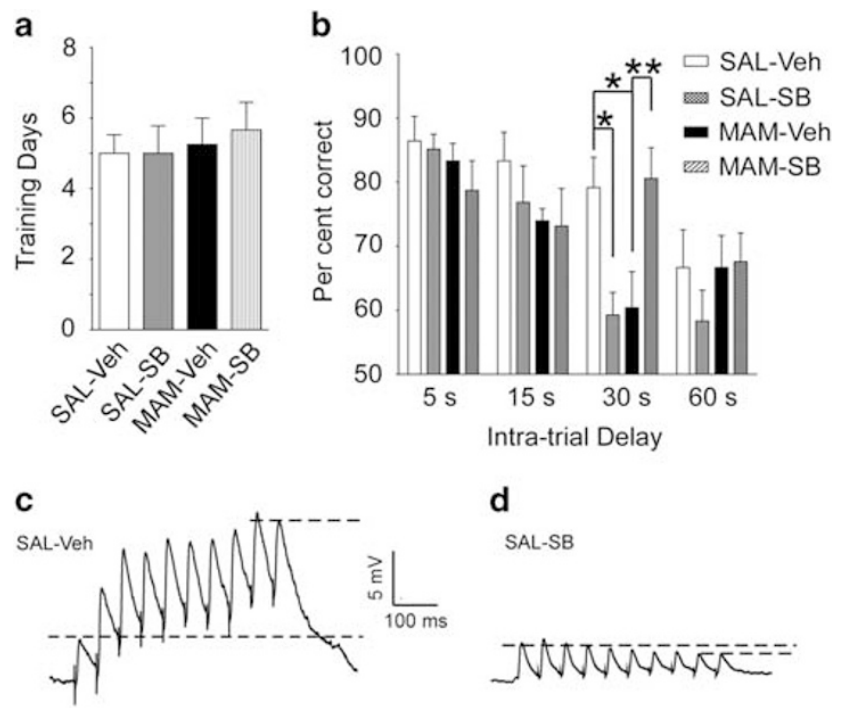

d

SAL-SB

e
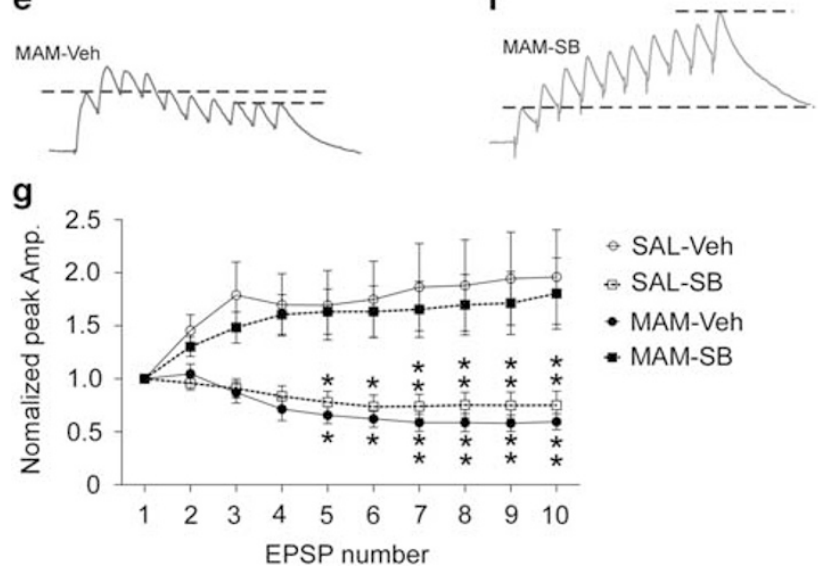

Figure 6 Treatment with glycogen synthase kinase-3 $\beta$ (GSK3 $\beta$ ) inhibitor (SB2 I 6763) during juvenile (P2 I-P28) improved working memory deficit in adult methylazoxymethanol (MAM) rats. (a) The required training days were comparable among different treatment groups: SAL-Veh $(n=8), \mathrm{SAL}-\mathrm{SB}$ $(n=9)$, MAM-Veh $(n=8)$, and MAM-SB $(n=9)$. (b) MAM-Veh and SAL-SB showed significant impairment in the test period with $30 \mathrm{~s}$ intra-trial delay. The four groups exhibited similar performance during other intra-trial delays. $* P<0.05$, ** $P<0.01$, RM-ANOVA. Two weeks later, adult SAL rats with juvenile SB216763 treatment showed reduced temporal summation of eEPSPs. (c-f) Representative traces of 10 stimuli evoked EPSPs from SALVeh $(n=12)$, SAL-SB $(n=12)$, MAM-Veh $(n=7)$, and MAM-SB $(n=9)$ groups. The SAL-Veh and MAM-SB groups showed increased EPSP summations, while the SAL-SB group exhibited reduced temporal summation, similar to that in MAM-Veh rats. (g) Summary graphs of the evoked EPSPs from different groups. ${ }^{*} P<0.05$, ** $P<0.0$ I vs SAL-Veh, RMANOVA followed by Bonferroni post hoc test. Veh, vehicle; MAM, methylazoxymethanol; SB, SB2 16763.

this is the first report of PFC dendritic spine reductions in this model. Moreover, GSK3 $\beta$ hyperactivity in the PFC occurred only during postnatal week 3 , a period of active spine growth and synaptogenesis in rodents (Kolb et al, 2012). During this critical developmental period, MAM rats have shown reduced pGSK3 $\beta$ Ser 9 in the PFC with an unaltered Akt activity. Since Akt is just one of the upstream kinases that are responsible for the Ser9 phosphorylation of GSK $3 \beta$, the hyperactive GSK $3 \beta$ could be mediated by an activation of protein phosphatase 1 (PP1) through dephosphorylation of GSK3 at the serine 9 residue. However, this assumption needs to be tested in the future.

GSK $3 \beta$ has long been implicated in the pathology of SZ (Kozlovsky et al, 2002; Lovestone et al, 2007). However, reports of GSK $3 \beta$ changes in SZ are contradictory. Our finding is consistent with a previous report of decreased pGSK3 $\beta$ Ser9 in the postmortem tissues from patients with SZ (Emamian et al, 2004), but differs from another study in which a decreased total protein level of GSK3 $\beta$ was reported (Kozlovsky et al, 2002). The reason for this discrepancy is unknown, but GSK3 $\beta$ levels in developing brain are likely different from those in postmortem brains of patients with $\mathrm{SZ}$, as reported in our study.

Although it is unlikely that GSK $3 \beta$ is the only protein altered throughout PFC development by prenatal MAM exposure, its leading role in regulating the interaction between LTP and LTD makes it a strong candidate in inducing aberrant neuroplastic processes that underlie early cognitive impairments. Indeed, GSK3 $\beta$ is involved in synaptic pruning via regulation of glutamate receptor cycling (Collingridge et al, 2010; Peineau et al, 2007). In a systematic investigation of $58 \mathrm{ser} / \mathrm{thr}$ protein kinases involved in NMDA receptor-dependent LTD, Peineau et al (2009)found evidence for the involvement of only GSK-3 $\beta$, but not other serine/threonine kinases in LTD induction. In support of this finding, our data showed that the transiently decreased pGSK $3 \beta$ level (ie hyperactive GSK $3 \beta$ ) in the MAM model may facilitate LTD induction in the PFC. Indeed, this conclusion was supported by several experimental results. First, the decrease of pGSK $3 \beta$ temporally correlated with a change in sensitivity for LTD induction in the juvenile MAM rat PFC. Second, inducing LTD via PP-LFS decreased pGSK $3 \beta$ level in the saline-treated rat PFC neurons. Third, the enhanced LTD induction in juvenile MAM rat PFC neurons could be effectively blocked by two structurally different GSK3 $\beta$ inhibitors. Fourth, in vivo treatment with GSK3 $\beta$ inhibitor during late juvenile period successfully rescued LTD change in MAM rats. The LTD induction in juvenile MAM animals was strikingly similar to that seen in the PFC of SAL-treated adolescent rats, suggesting that a transient, normally occurring developmental window for synaptic LTD induction in the adolescent PFC significantly advanced to the juvenile stage by gestational MAM exposure and the resulted increase in GSK3 $\beta$ activity, as illustrated in Supplementary Figure S8.

In addition to LTD facilitation, we also found a deficit in LTP induction that may be mediated by the hyperactive GSK $3 \beta$, consistent with our previous report in the hippocampus (Snyder et al, 2013). Indeed, activation of GSK3 $\beta$ suppressed LTP induction in the hippocampus (Zhu et al, 2007). Both transgenic mice overexpressing GSK3 $\beta$ and Fmr1 knockout mice with decreased pGSK3 $\beta$ Ser9 in dentate gyrus also showed a reduced LTP (Franklin et al, 2014; Hooper et al, 2007).

GSK $3 \beta$ is located in both pre- and postsynaptic sites (Peineau et al, 2007; Zhu et al, 2007). LTD facilitation often reflects a decrease in the magnitude of evoked synaptic responses due to a persistent decrease in presynaptic transmitter release and/or postsynaptic AMPA receptor trafficking and phosphorylation (Collingridge et al, 2010). A recent study also reported that activation of GSK $3 \beta$ inhibits presynaptic vesicle exocytosis by phosphorylating 
P/Q-type calcium channels and interrupting SNARE complex formation (Zhu et al, 2010). However, our data suggest that the facilitated LTD induction involves a postsynaptic mechanism. First, the PPRs were not changed in the evoked EPSCs, indicating unaltered presynaptic release of vesicles. Second, the facilitated LTD induction was completely blocked when the recording pipette was loaded with GSK3 $\beta$ inhibitor. The increased GSK3 $\beta$ activity seems to be required for AMPA receptor internalization and LTD induction through the phosphorylation and destabilization of PSD-95 in the postsynaptic density (Nelson et al, 2013). It is possible that activation of GSK3 $\beta$ directly interacts with AMPA receptor subunits and causes internalization of AMPA receptors during activity-dependent synaptic plasticity, as demonstrated in Supplementary Figure S8 (Peineau et al, 2007). In fact, GSK3 $\beta$, which is also increased in depression, can be activated by PP1 and thus contributes to synaptic destabilization by promoting the internalization of GluA1.

One of the most replicated findings in SZ research is reduced dendritic spine density of pyramidal neurons in the PFC that may occur during early postnatal development (Broadbelt et al, 2002; Glantz and Lewis, 2000; Penzes et al, 2011). Previous studies have shown that potentiation of individual spine synapses selectively increases their stability, resulting in spine survival (De Roo et al, 2008). Conversely, LTD leads to homogeneous depression of synaptic function, followed by selective removal of weakly integrated synapses (Wiegert and Oertner, 2013). In juvenile MAM rats, transiently hyperactive GSK $3 \beta$ impairs LTP and facilitates LTD, which in turn promotes synaptic pruning and spine elimination during the critical period for cortical development. Importantly, the rescue of LTP/LTD deficits by GSK3 $\beta$ inhibition suggests that targeting GSK3 $\beta$ during the presymptomatic stage of SZ could ameliorate eventual spine deficits and cognitive symptoms in adulthood. Indeed, we found that the reduced spine density in apical dendritic and working memory deficits in MAM rats were effectively rescued by administration of a GSK3 $\beta$ inhibitor during the late juvenile period (P21-P28), suggesting that GSK3 $\beta$ inhibition reversed these neuroplasticity deficits by normalizing activity-dependent synaptic plasticity. One would argue that this study is limited by relying on a single animal model of SZ, but our finding is in agreement with a very recent study in which treatment of GSK3 inhibitor during early postnatal development also rescues neurophysiological and behavioral deficits in the 22q11.1 microdeletion model of SZ (Tamura et al, 2016).

It has been reported that GSK3 $\beta$ inhibition exhibits a cognitive-enhancing effect on a broad spectrum of neuropsychiatric disorders, making it a promising target for cognitive deficits (King et al, 2014); indeed, it works well for the MAM-exposed animals. However, we have also realized a problem with the treatment of SAL control animals. Although juvenile treatment with GSK3 $\beta$ inhibitor shows benefits in MAM rats, adult SAL rats that received juvenile SB216763 treatment also exhibit obvious deficits in working memory performance, similar to the memory impairment observed in the MAM rats with vehicle treatment. To confirm our observation and to clarify the mechanism, we examined the temporal summation of EPSPs in prefrontal cortical pyramidal neurons. Interestingly, we found deficits in temporal summation of EPSPs in response to a 10-pulse train stimulus both in SAL rats with SB exposure and MAM rats with vehicle exposure. Specifically, the synaptic transmission in the SAL-SB rat PFC pyramidal neurons displayed a reduced temporal summation, similar to that in the MAM-Veh group. Because persistent firing of cortical neurons in response to a transient stimulus is an attractive mechanism for short-term information storage, the blunted EPSP summation observed in SAL-SB rats may reflect the functional deficits in working memory.

Moreover, we also found that although the decreased spine density in MAM rats can be recovered by treatment with GSK3 inhibitor during the juvenile period, the adult SAL rats with SB treatment also exhibit changes in dendritic spine morphology. Specifically, we observed a significant decrease of mushroom spine and increase of stubby spine in SAL-SB in apical dendrites of layer $\mathrm{V}$ pyramidal neurons compared with those in SAL rats with vehicle. This finding is consistent with a recent study in which inducible knockout of GSK3 $\beta$ in cortical and hippocampal neurons induces an increase of stubby spines and a reduction of mushroom spines (Ochs et al, 2015). Although the exact functional correlates of spine morphology remain unknown, mushroom spine represents functional maturation of synaptic connection (Sala and Segal, 2014). Studies have also implicated mushroom spines in memory (Bourne and Harris, 2007; Matsuo et al, 2008), and indeed, spatial and working memory is linked to spine density and mushroom spines (Mahmmoud et al, 2015). The reduction of mushroom spines in adult SAL-SB rats could thus explain the working memory impairment although how brief exposure of GSK3 inhibitor during early postnatal development leads to long-lasting disruption of spine maturation remains to be determined.

We propose that developmental inhibition of GSK3 $\beta$ in normal PFC results in delayed spine maturation, as supported by the increased immature (stubby) spines and decreased mature (mushroom) spines in the mPFC of SAL-SB rats. Despite no spine density changes in SAL-SB rats, the altered mature shape of spines in these animals probably reflects different spine morphology alterations from that in MAM rats (decreased spine density) with a similar working memory performance. We hypothesize that hypoactive GSK3 $\beta$ in developing PFC is associated with immature spine shape, while hyperactive GSK3 $\beta$ links to spine overgrowth/pruning. Mechanically, we speculate that GSK3 $\beta$ inhibition regulates spines via GluN2B subunit because downregulation of cortical GluNR2B subunit-containing NMDARs is required for spine maturation. Nevertheless, our results indicate that although treatment with GSK3 inhibitor displays significant benefits in MAM animals, it should be applied cautiously to animals without an abnormal GSK3 activity. In fact, potential detrimental effect of chronic GSK3 inhibitor exposure on cognitive functions has been reported (Stip et al, 2000; Wingo et al, 2009).

In conclusion, we report here that an increased GSK3 $\beta$ activity in early development directly contributes to altered synaptic plasticity, spine loss, and cognitive disability in a neurodevelopmental model of SZ. Although inhibition of GSK3 $\beta$ activity is emerging as a promising therapeutic option for cognitive impairments in multiple neuropsychiatric disorders, early treatment should be emphasized for SZ intervention due to the limited window of GSK3 $\beta$ hyperactivity during 
development. The findings described here may shed light on a feasible early therapeutic approach for SZ.

\section{FUNDING AND DISCLOSURE}

Funding for the current study was provided by the NIH R01MH085666 to WJG. The authors declare no conflict of interest.

\section{ACKNOWLEDGMENTS}

We thank Miss Yelena Gulchina and Miss Sarah Monaco for their thoughtful comments and editorial work. BX designed and carried out all experiments and data analysis of western blots, electrophysiological recordings, morphological analysis, and behavioral tests. He wrote the paper. YCL helped electrophysiological recording and read the manuscript. WJG conceived the study, supervised the project, and finalized the manuscript.

\section{REFERENCES}

Bastrikova N, Gardner GA, Reece JM, Jeromin A, Dudek SM (2008). Synapse elimination accompanies functional plasticity in hippocampal neurons. Proc Natl Acad Sci USA 105: 3123-3127.

Beurel E, Mines MA, Song L, Jope RS (2012). Glycogen synthase kinase-3 levels and phosphorylation undergo large fluctuations in mouse brain during development. Bipolar Disord 14: 822-830.

Bourgeois JP, Goldman-Rakic PS, Rakic P (1994). Synaptogenesis in the prefrontal cortex of rhesus monkeys. Cereb Cortex 4: 78-96.

Bourne J, Harris KM (2007). Do thin spines learn to be mushroom spines that remember? Curr Opin Neurobiol 17: 381-386.

Broadbelt K, Byne W, Jones LB (2002). Evidence for a decrease in basilar dendrites of pyramidal cells in schizophrenic medial prefrontal cortex. Schizophr Res 58: 75-81.

Carpenter WT, Koenig JI (2008). The evolution of drug development in schizophrenia: past issues and future opportunities. Neuropsychopharmacology 33: 2061-2079.

Caruana DA, Warburton EC, Bashir ZI (2011). Induction of activity-dependent LTD requires muscarinic receptor activation in medial prefrontal cortex. J Neurosci 31: 18464-18478.

Collingridge GL, Peineau S, Howland JG, Wang YT (2010). Longterm depression in the CNS. Nat Rev Neurosci 11: 459-473.

Cuesto G, Enriquez-Barreto L, Carames C, Cantarero M, Gasull X, Sandi C et al (2011). Phosphoinositide-3-kinase activation controls synaptogenesis and spinogenesis in hippocampal neurons. J Neurosci 31: 2721-2733.

De Roo M, Klauser P, Muller D (2008). LTP promotes a selective long-term stabilization and clustering of dendritic spines. PLoS Biol 6: e219.

Emamian ES, Hall D, Birnbaum MJ, Karayiorgou M, Gogos JA (2004). Convergent evidence for impaired AKT1-GSK3beta signaling in schizophrenia. Nat Genet 36: 131-137.

Flagstad P, Glenthoj BY, Didriksen M (2005). Cognitive deficits caused by late gestational disruption of neurogenesis in rats: a preclinical model of schizophrenia. Neuropsychopharmacology 30: $250-260$

Franklin AV, King MK, Palomo V, Martinez A, McMahon LL, Jope RS (2014). Glycogen synthase kinase-3 inhibitors reverse deficits in long-term potentiation and cognition in fragile $\mathrm{X}$ mice. Biol Psychiatry 75: 198-206.

Freyberg Z, Ferrando SJ, Javitch JA (2010). Roles of the Akt/GSK-3 and Wnt signaling pathways in schizophrenia and antipsychotic drug action. Am J Psychiatry 167: 388-396.
Glantz LA, Lewis DA (2000). Decreased dendritic spine density on prefrontal cortical pyramidal neurons in schizophrenia. Arch Gen Psychiatry 57: 65-73.

Glausier JR, Lewis DA (2013). Dendritic spine pathology in schizophrenia. Neuroscience 251: 90-107.

Hooper C, Markevich V, Plattner F, Killick R, Schofield E, Engel T et al (2007). Glycogen synthase kinase-3 inhibition is integral to long-term potentiation. Eur J Neurosci 25: 81-86.

Insel TR (2010). Rethinking schizophrenia. Nature 468: 187-193.

King MK, Pardo M, Cheng Y, Downey K, Jope RS, Beurel E (2014). Glycogen synthase kinase-3 inhibitors: rescuers of cognitive impairments. Pharmacol Ther 141: 1-12.

Kolb B, Mychasiuk R, Muhammad A, Li Y, Frost DO, Gibb R (2012). Experience and the developing prefrontal cortex. Proc Natl Acad Sci USA 109 (Suppl 2): 17186-17193.

Kozlovsky N, Belmaker RH, Agam G (2002). GSK-3 and the neurodevelopmental hypothesis of schizophrenia. Eur Neuropsychopharmacol 12: 13-25.

Leroy K, Brion JP (1999). Developmental expression and localization of glycogen synthase kinase-3beta in rat brain. J Chem Neuroanat 16: 279-293.

Li X, Rosborough KM, Friedman AB, Zhu W, Roth KA (2007). Regulation of mouse brain glycogen synthase kinase- 3 by atypical antipsychotics. Int J Neuropsychopharmacol 10: 7-19.

Li YC, Xi D, Roman J, Huang YQ, Gao WJ (2009). Activation of glycogen synthase kinase-3 beta is required for hyperdopamine and D2 receptor-mediated inhibition of synaptic NMDA receptor function in the rat prefrontal cortex. J Neurosci 29: 15551-15563.

Lodge DJ, Grace AA (2009). Gestational methylazoxymethanol acetate administration: a developmental disruption model of schizophrenia. Behav Brain Res 204: 306-312.

Lovestone S, Killick R, Di Forti M, Murray R (2007). Schizophrenia as a GSK-3 dysregulation disorder. Trends Neurosci 30: 142-149.

Mahmmoud RR, Sase S, Aher YD, Sase A, Groger M, Mokhtar M et al (2015). Spatial and working memory is linked to spine density and mushroom spines. PLoS One 10: e0139739.

Matsuo N, Reijmers L, Mayford M (2008). Spine-type-specific recruitment of newly synthesized AMPA receptors with learning. Science 319: 1104-1107.

Nadri C, Lipska BK, Kozlovsky N, Weinberger DR, Belmaker RH, Agam G (2003). Glycogen synthase kinase (GSK)-3beta levels and activity in a neurodevelopmental rat model of schizophrenia. Brain Res Dev Brain Res 141: 33-37.

Nagerl UV, Eberhorn N, Cambridge SB, Bonhoeffer T (2004). Bidirectional activity-dependent morphological plasticity in hippocampal neurons. Neuron 44: 759-767.

Nelson CD, Kim MJ, Hsin H, Chen Y, Sheng M (2013). Phosphorylation of threonine-19 of PSD-95 by GSK-3beta is required for PSD-95 mobilization and long-term depression. J Neurosci 33: 12122-12135.

Ochs SM, Dorostkar MM, Aramuni G, Schon C, Filser S, Poschl J et al (2015). Loss of neuronal GSK3beta reduces dendritic spine stability and attenuates excitatory synaptic transmission via betacatenin. Mol Psychiatry 20: 482-489.

Peineau S, Nicolas CS, Bortolotto ZA, Bhat RV, Ryves WJ, Harwood AJ et al (2009). A systematic investigation of the protein kinases involved in NMDA receptor-dependent LTD: evidence for a role of GSK-3 but not other serine/threonine kinases. Mol Brain 2: 22.

Peineau S, Taghibiglou C, Bradley C, Wong TP, Liu L, Lu J et al (2007). LTP inhibits LTD in the hippocampus via regulation of GSK3beta. Neuron 53: 703-717.

Penzes P, Cahill ME, Jones KA, VanLeeuwen JE, Woolfrey KM (2011). Dendritic spine pathology in neuropsychiatric disorders. Nat Neurosci 14: 285-293.

Peters A, Kaiserman-Abramof IR (1970). The small pyramidal neuron of the rat cerebral cortex. The perikaryon, dendrites and spines. Am J Anat 127: 321-355.

Sala C, Segal M (2014). Dendritic spines: the locus of structural and functional plasticity. Physiol Rev 94: 141-188. 
Sanderson TM, Cotel MC, O'Neill MJ, Tricklebank MD, Collingridge GL, Sher E (2012). Alterations in hippocampal excitability, synaptic transmission and synaptic plasticity in a neurodevelopmental model of schizophrenia. Neuropharmacology 62: $1349-1358$.

Selemon LD (2013). A role for synaptic plasticity in the adolescent development of executive function. Transl Psychiatry 3: e238.

Snyder MA, Adelman AE, Gao WJ (2013). Gestational methylazoxymethanol exposure leads to NMDAR dysfunction in hippocampus during early development and lasting deficits in learning. Neuropsychopharmacology 38: 328-340.

Spear LP (2000). The adolescent brain and age-related behavioral manifestations. Neurosci Biobehav Rev 24: 417-463.

Stip E, Dufresne J, Lussier I, Yatham L (2000). A double-blind, placebo-controlled study of the effects of lithium on cognition in healthy subjects: mild and selective effects on learning. $J$ Affect Disord 60: 147-157.

Tamura M, Mukai J, Gordon JA, Gogos JA (2016). Developmental inhibition of Gsk3 rescues behavioral and neurophysiological deficits in a mouse model of schizophrenia predisposition. Neuron 89: 1100-1109.

Toyoda H, Zhao MG, Zhuo M (2005). Roles of NMDA receptor NR2A and NR2B subtypes for long-term depression in the anterior cingulate cortex. Eur J Neurosci 22: 485-494.
Wang HX, Gao WJ (2009). Cell type-specific development of NMDA receptors in the interneurons of rat prefrontal cortex. Neuropsychopharmacology 34: 2028-2040.

Wiegert JS, Oertner TG (2013). Long-term depression triggers the selective elimination of weakly integrated synapses. Proc Natl Acad Sci USA 110: E4510-E4519.

Wingo AP, Wingo TS, Harvey PD, Baldessarini RJ (2009). Effects of lithium on cognitive performance: a meta-analysis. J Clin Psychiatry 70: 1588-1597.

Xi D, Li YC, Snyder MA, Gao RY, Adelman AE, Zhang W et al (2011). Group II metabotropic glutamate receptor agonist ameliorates MK801-induced dysfunction of NMDA receptors via the Akt/GSK-3beta pathway in adult rat prefrontal cortex. Neuropsychopharmacology 36: 1260-1274.

Zhou Q, Homma KJ, Poo MM (2004). Shrinkage of dendritic spines associated with long-term depression of hippocampal synapses. Neuron 44: 749-757.

Zhu LQ, Liu D, Hu J, Cheng J, Wang SH, Wang Q et al (2010). GSK-3 beta inhibits presynaptic vesicle exocytosis by phosphorylating P/Q-type calcium channel and interrupting SNARE complex formation. J Neurosci 30: 3624-3633.

Zhu LQ, Wang SH, Liu D, Yin YY, Tian Q, Wang XC et al (2007). Activation of glycogen synthase kinase-3 inhibits long-term potentiation with synapse-associated impairments. J Neurosci 27: 12211-12220.

Supplementary Information accompanies the paper on the Neuropsychopharmacology website (http://www.nature.com/npp) 